\title{
Testicular apoptosis in feral Clarias gariepinus using TUNEL and cleaved caspase- 3 immunohistochemistry
}

\author{
Leon M. McClusky ${ }^{\mathrm{a}}$, Irene E.J. Barnhoorn ${ }^{\mathrm{b}}$, Jacobus C. van Dyk ${ }^{\mathrm{b}}$ and Maria S. \\ Bornman $^{\mathrm{b}}$ \\ ${ }^{a}$ Department of Physiology, Faculty of Health Sciences, University of Pretoria, Pretoria, \\ South Africa \\ ${ }^{b}$ Department of Urology, Faculty of Health Sciences, University of Pretoria, Pretoria, \\ South Africa
}

\begin{abstract}
This paper reports on the mechanistic basis of cellular death in the testis of Clarias gariepinus using the TUNEL and caspase-3 immunohistochemistry. It was also aimed to determine the testicular zone most suitable for the quantification of testicular apoptosis. The results showed that based on its immuno-expression patterns, activated caspase-3 has a clear and defined role in the progression of germ cell apoptosis in spermatogenically active catfish testis. Caspase-3 activation, and not TUNEL-detected DNA fragmentation, is associated with condensation of chromatin into a single mass. Testes of spermatogenically active catfish consist of spermatogenic, mature and spent tubules. Spermatogenic tubules were concluded to be the most suitable zone for the quantification of testicular cell death ratios as apoptotic events occurred predominantly in the secondary spermatocytes. The findings of this study will form the basis to link apoptotic events in the testes of C. gariepinus with the effects of EDC exposure in future studies.
\end{abstract}

Keywords: Apoptosis; Spermatocytes; TUNEL; Caspase-3 immunohistochemistry; Sharptooth catfish; Clarias gariepinus

\section{Introduction}

Clarias gariepinus is a resilient bottom-dwelling indigenous African teleost species, whose testicular steroid biosynthesis activities are also well described (Schulz et al., 1996; [Cavaco et al., 1997] and [Cavaco et al., 1998]). The testes of species such as $C$. gariepinus and other amniotes are also useful model systems to study toxicant actions stage-by-stage during spermatogenesis. They are organisationally less complex than those of mammals and other amniotes since spermatogenesis proceeds in a cystic mode (a germ cell clone in any given stage is associated with Sertoli cells, in a discrete follicle-like unit, the spermatocyst). This anatomical arrangement further indicates that the various germ cell stages have different hormonal requirements and may therefore have different sensitivities to any given chemical. Since all germ cells in a cyst are syncitially connected, it is expected that the spread of a death signal would be rapid and that clone death would be all-or-none. To determine whether exposure to environmental toxicants 
will increase testicular apoptosis in a sentinel species like C. gariepinus, the mechanistic basis of cellular death of this species first needs to be investigated.

C. gariepinus is an abundant species in the Rietvlei Dam (RVD), situated within the Rietvlei Nature Reserve (RNR) located near a large metropolitan area in South Africa. The RVD receives effluent from sewage and industrial plants (Barnhoorn et al., 2004).

Subsequently, the testes of C. gariepinus inhabiting the RVD has been intensively studied regarding reproductive and developmental abnormalities after exposure to endocrine disruptor chemicals (EDCs) (Barnhoorn et al., 2004). However, testicular apoptosis have not yet been described in this sentinel species. To link apoptotic events in the testes of $C$. gariepinus with the effects of EDC exposure, the methodology for determining the mechanistic basis of cellular death first needs to be tested.

The detection of fragmented DNA with in situ end-labelling techniques, such as the terminal transferase-mediated dUTP nick-end-labelling assay (TUNEL), facilitates the quantification of the actual numbers of germinal clones that will be deleted via apoptosis from the spermatogenic progression ([McClusky, 2005] and [McClusky, 2006]). Recent advances have made possible the immunohistochemical detection of earlier phases of apoptosis such as the activation of cysteine aspartyl-specific proteases (caspases), including cleaved caspase- 3 which is a major executioner of apoptotic morphology (Gown and Willingham, 2002). Furthermore, unlike the situation in mammals, testicular apoptosis in fish is prolonged and may take 10 months to complete in the case of cartilaginous fishes (McClusky, 2005).

The aims of this study were to (1) determine the mechanistic basis of cellular death in the testis of C. gariepinus using the TUNEL and caspase-3 immunohistochemistry and (2) to identify the most suitable testicular zone of spermatogenic activity for the quantification of testicular apoptosis.

\section{Materials and methods}

The protocol was approved by the Animal Use and Care Committee of the University of Pretoria.

\subsection{Test organisms and tissue sampling}

Sexually mature adult feral C. gariepinus, $(n=11 ; 2.5-5.0 \mathrm{~kg} ; 67-87 \mathrm{~cm}$ total body length) were captured during their spawning season (midsummer) from the RVD (Barnhoorn et al., 2004). Only adult fish with a body mass above $2.5 \mathrm{~kg}$ were sampled. The histological evaluation confirmed that all the fish were sexually mature.

The fish were sacrificed by severing the spinal cord. The testes were excised and samples taken for histology using the salient morphological features of the testis of $C$. gariepinus previously described by Cavaco et al. (1998). In brief, the paired elongated and relatively flattened testis is medially attached to a connective tissue rich central portion, which also contains the spermatid duct. Although the spermatogenically active catfish testis has a 
simple medial to lateral zonation, individual members of the fish population caught at any given time in the spawning season are not all synchronised in terms of their testicular development and testicular zonation may vary between specimens. Thus, the zone containing the spermatogenic tubules include tubules that are lined by single primary spermatogonia, primary and secondary spermatocyte, and early spermatid, while the lumen is partially filled with spermatozoa. Mature tubules are fully distended with spermatozoa, and are lined with a scattering of single spermatogonia and some spermatocyte cysts. Spent tubules are largely devoid of spermatozoa, and lined with Sertoli cell remnants and the occasional spermatogonium. Thus, spermatogenic, mature and spent tubules can be present within the same specimen.

\subsection{Tissue preparation}

Midsections of the testes $(25 \mathrm{~mm} \times 15 \mathrm{~mm})$ were sampled and fixed in $10 \%$ neutral buffered formalin in phosphate buffered saline (PBS) for $72 \mathrm{~h}$, after which they were embedded in paraplast. Sections of $4 \mu \mathrm{m}$ thick were floated onto a distilled water bath $\left(45^{\circ} \mathrm{C}\right)$, collected on superfrost plus slides, deparaffinised and rehydrated stepwise through an ethanol series and processed for routine histological analysis using haematoxylin and eosin staining (HE) and immunohistochemistry.

\subsection{TUNEL immunohistochemistry}

Deparaffinised and rehydrated sections were subjected to a pre-treatment protocol as described in Labat-Moleur et al. (1998), which involved microwave oven treatment $(800 \mathrm{~W})$ in $10 \mathrm{mM}$ citrate buffer $(\mathrm{pH} 3.0)$ for $14 \mathrm{~min}$. Following treatment for $2 \mathrm{~min}$ in 0.1 Triton X-100 in $0.1 \%$ sodium citrate on ice. Sections were treated with proteinase K $(20 \mu \mathrm{g} / \mathrm{mL})$ for $15 \mathrm{~min}$ at room temperature (RT), after which they were washed twice with PBS-A (50 mM sodium phosphate, $\mathrm{pH} 7.4 ; 200 \mathrm{mM} \mathrm{NaCl}$ ). Endogenous peroxidase was reduced by treating sections in darkness with $3 \%$ hydrogen peroxide in PBS-A for 5 min, followed by two washes in PBS-A. Subsequent steps for TUNEL staining were carried out using the ApopTag-Peroxidase Kit according to the supplier's instructions (Chemicon, USA). Following incubation of the sections with the TUNEL reaction mixture in a humidified chamber at $37^{\circ} \mathrm{C}$ for $1 \mathrm{~h}$, antidigoxigenin-peroxidase complex was added for $30 \mathrm{~min}$ at RT. Sections were then treated with 3,3'-diaminobenzidine (DAB) for 1-2 min according to the supplier's instructions (Vectorlabs, Burlingame, CA, USA) such that positive cells stained brown. Sections were counterstained with $0.5 \%$ crystal violet-containing methyl green in $100 \mathrm{mM}$ sodium acetate $(\mathrm{pH} \mathrm{4.0)}$, dehydrated in $100 \%$ butanol, cleared in xylene and mounted with Entellan (Merck, South Africa). To generate negative controls, terminal deoxynucleotidyl transferase (TdT) was replaced with sterile water, whereas for positive controls, sections were pre-treated with DNase I $(10 \mu \mathrm{g} / \mathrm{mL}$ in distilled water) to generate DNA fragments.

\subsection{Cleaved caspase-3 immunohistochemistry}

Deparaffinised and rehydrated sections were subjected to an antigen retrieval procedure by heating the sections in a microwave oven $(800 \mathrm{~W})$ in $10 \mathrm{mM}$ sodium citrate buffer $(\mathrm{pH}$ 
6.0) for $10 \mathrm{~min}$. After cooling for $20 \mathrm{~min}$ at RT, sections were rinsed in distilled water and treated in darkness for 10 min with $3 \%$ hydrogen peroxide to reduced endogenous peroxidase. Following a rinse in distilled water, the sections were placed in $0.1 \%$ Tween 20 in PBS-B (pH 7.4) for 5 min. Sections were then incubated with blocking solution (5\% normal goat serum in $0.1 \%$ Tween 20 -PBS-B) for $1 \mathrm{~h}$ at RT to reduce non-specific binding, and then overnight at $4{ }^{\circ} \mathrm{C}$ with a rabbit polyclonal antibody to cleaved caspase3 (17/19 kDa fragment of activated caspase-3; Cell Signaling Technology, Beverly, MA, USA) diluted 1:100 in blocking solution. After three washes in 0.1\% Tween 20-PBS-B, sections were incubated with biotinylated antirabbit IgG for $30 \mathrm{~min}$ at RT, washed in PBS-B, and then incubated with the Vectastain avidin-biotin complex (Vectorlabs) for a further $30 \mathrm{~min}$ at RT. Following three 5 min PBS-B washes, the antigen was finally detected by treating the sections with 3,3'-diaminobenzidine, counterstained, dehydrated, cleared and mounted as described in the previous section for the TUNEL immunohistochemistry. Negative controls were generated by omitting the primary antibody and by serial dilution of the primary antibody, as well as by using a rabbit monoclonal antibody to cleaved caspase-3. The rat testicular sections used as positive controls were those from a previous study (McClusky et al., 2007).

\subsection{Microscopy}

Quantitative measurements were done on a Nikon Optiphot microscope fitted with an ocular grid in one eyepiece. For each testicular zone and using a $10 \times$ objective, each one of the 50 tubules intersecting each of two widely separated lines parallel to the adjacent testicular zone were scored for the number of caspase-3-positive and TUNEL-positive cells it contained, and the average number was taken of the two lines. For the spermatogenic tubule zone and using a $20 \times$ objective, each one of a 100 randomly counted spermatogenic tubules were scored as caspase-3-postive or -negative. A tubule was designated caspase-3-positive if it contained at least one cluster of four caspase-3postive cells. Sections were photographed with a Nikon Optiphot microscope, fitted with a Nikon DXM1200F digital camera connected to a PC equipped with image-capturing software.

\subsection{Statistics}

The data were expressed as the mean number of caspase-3-positive and TUNEL-positive cells per 50 tubules in each testicular zone, and the mean number of caspase-3-positive tubules per 100 spermatogenic tubules. Data were examined by two-way ANOVA using Instat Version 2.03 (GraphPad Software, San Diego, CA, USA). Tukey's multiplecomparisons test was used to determine which values differed significantly $(p<0.05)$.

\section{Results}

The histological evaluation of the testes showed that the spermatogenic tubules contained spermatocysts in various stages of spermatogenic development (Fig. 1A). Analysis of routine HE-stained sections showed degenerated cells scattered in mainly spermatocyte cysts in spermatogenically active fish. These were single cells with either irregularly 
shaped intensely basophilic (Fig. 1B) or pyknotic nuclei (Fig. 1C), and, more rarely, groups of combined germ cells with characteristic horse-shoe shaped marginated chromatin (Fig. 1B). Besides the occasional dying spermatogonium (not shown), most dying cells were primary spermatocytes and more frequently secondary spermatocytes (Fig. 1B and C), but never testicular somatic cells (Sertoli cells, Leydig cells, and peritubular cells). These results suggest that cell death, some features of which have apoptosis-like morphology, may be an integral component of spermatogenetic activity in catfish.
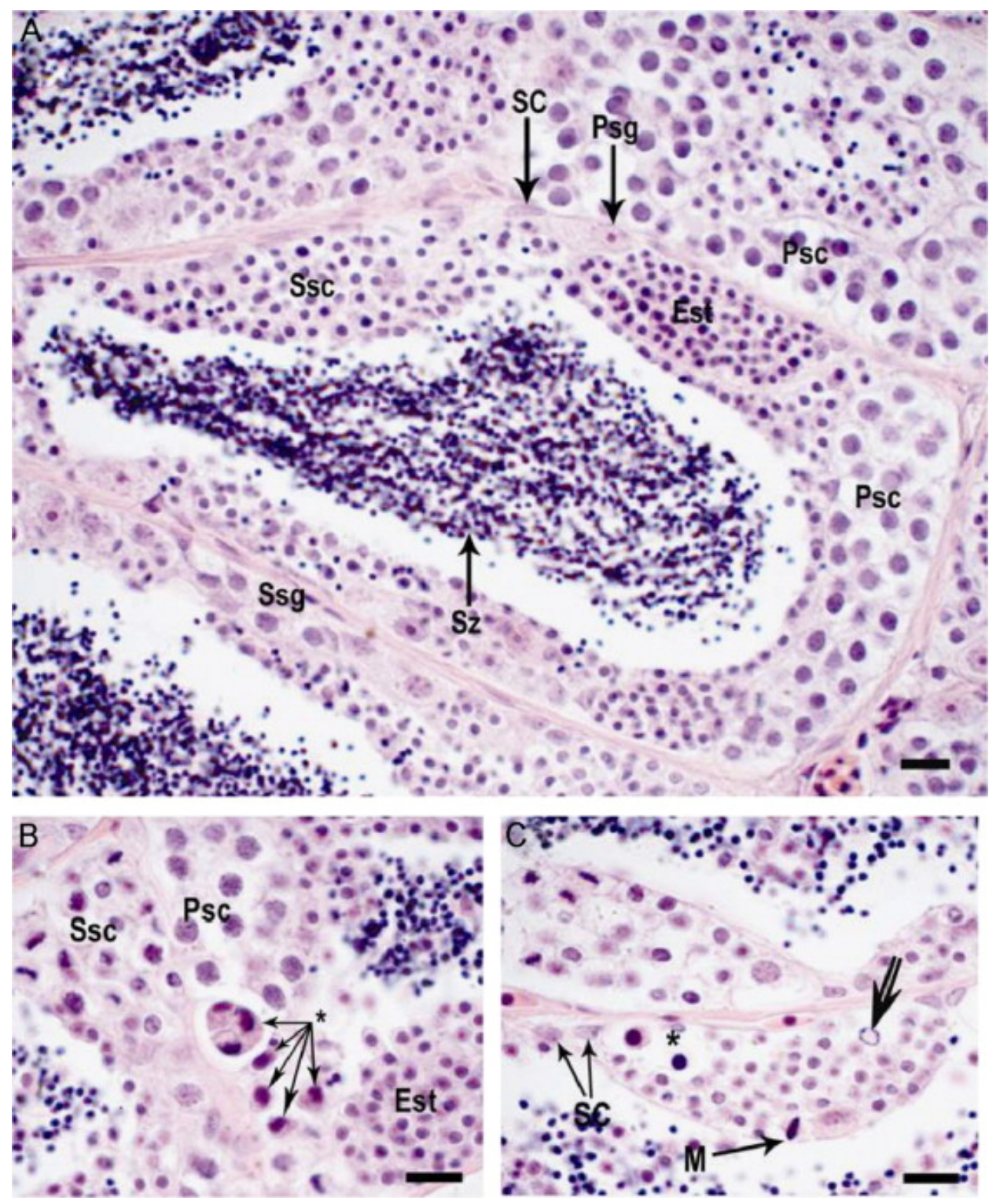

Fig. 1. Low $(A)$ and high $(B, C)$ magnification photomicrographs of haematoxylin and eosin-stained testicular sections of catfish. (A) Spermatogenic tubules contained the complete spermatogenic sequence and were lined by numerous spermatocysts that each contained a different germ cell stage. The spermatozoa (sz) were gathered in the tubule lumen. Degenerate cells (*) were found scattered mainly in spermatocyte cysts, and 
included single cells with either irregularly shaped intensely basophilic nuclei (B), or with pyknotic nuclei $(C)$, or with chromatin arranged in a ring-like manner in the nucleus (open arrow, $C$ ), and, groups of coalesced cells with horse-shoe shaped condensed chromatin (B). Degenerate cells were occasionally coincident with mitotically active (M) cells in the same cyst (C). Psg, primary spermatogonia; Ssg, secondary spermatogonia; Psc, primary spermatocytes; Ssc, secondary spermatocytes; Est, early spermatids; SC, Sertoli cell nuclei. Bar $=10 \mu \mathrm{m}$.

TUNEL-labelling was variable, with combined germ cell masses only partially TUNELlabelled in some instances, whereas they were completely TUNEL-labelled in other instances (Fig. 2A). Likewise, degenerate-looking germ cells with intensely blue-stained nuclei similar in colour to condensed chromatin in the heads of adjacent spermatozoa were conspicuously TUNEL-negative (Fig. 2B). All cell types were, however, labelled after DNase I treatment of tissue sections (not shown), evidence that the observed negative labelling was actually due to the absence of fragmented DNA rather than a lack of access of TUNEL reagents in situ.
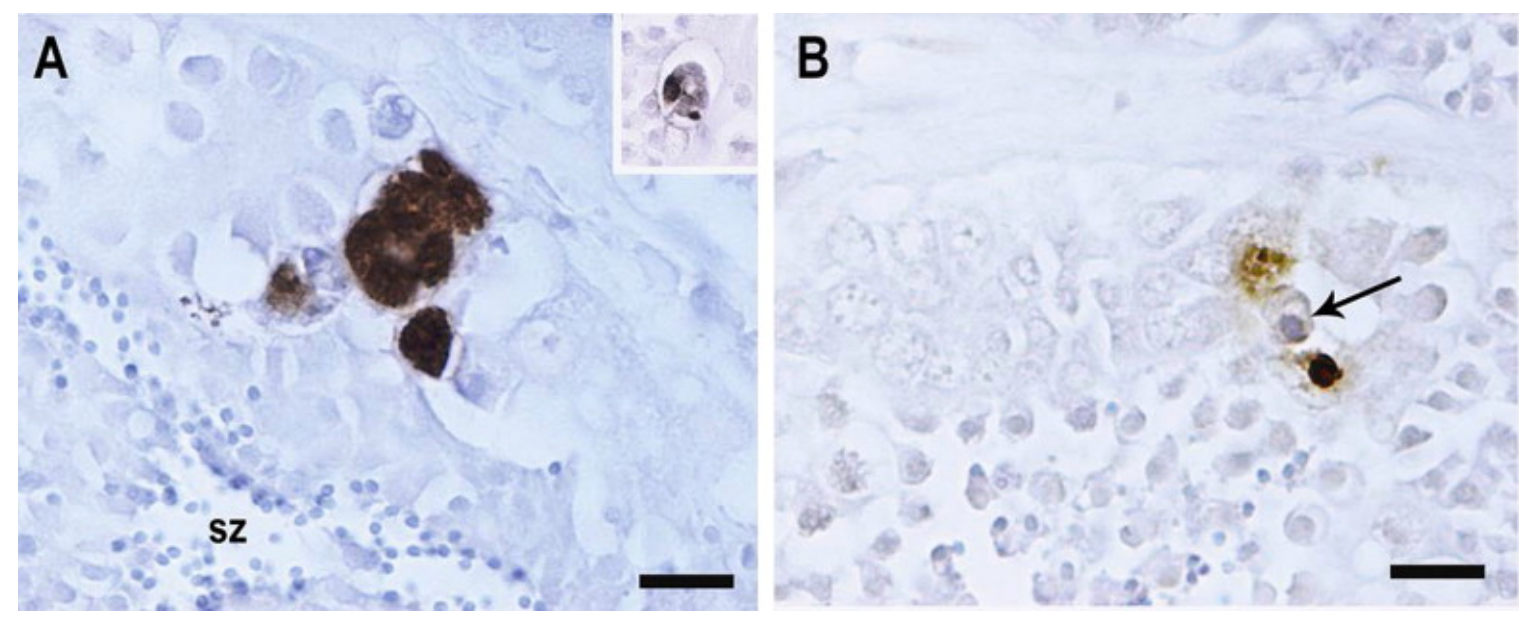

Fig. 2. Photomicrographs of TUNEL-stained testicular sections of RVD catfish. (A) Coalesced germ cell masses were either completely or partially TUNEL-labelled (inset). (B) Some cysts only contained one or two TUNEL-labelled cells. Note TUNEL-negative degenerate-looking cell with intensely blue-stained nuclei similar in color to spermatozoa (arrow); sz, spermatozoa. Bar $=10 \mu m$.

Given this apparent selectivity of the TUNEL method, which labels the end-stage of apoptosis, such as the fragmented DNA, we wished to determine immunohistochemically whether upstream apoptotic events could be labelled. Cleaved caspase-3 immunohistochemistry was performed on the testes of RVD catfish. Testicular sections 
of $p$-NP treated rats were simultaneously processed and served as positive controls. Unlike with TUNEL, cleaved caspase-3 immunoreactivity was very common in the spermatogenically active RVD catfish (Fig. 3A). Labelling was strong and specific, limited to germ cells and was found in scattered isolated cells (Fig. 3A), groups of cells (Fig. 3A), and groups of combined germ cells (Fig. 3B).
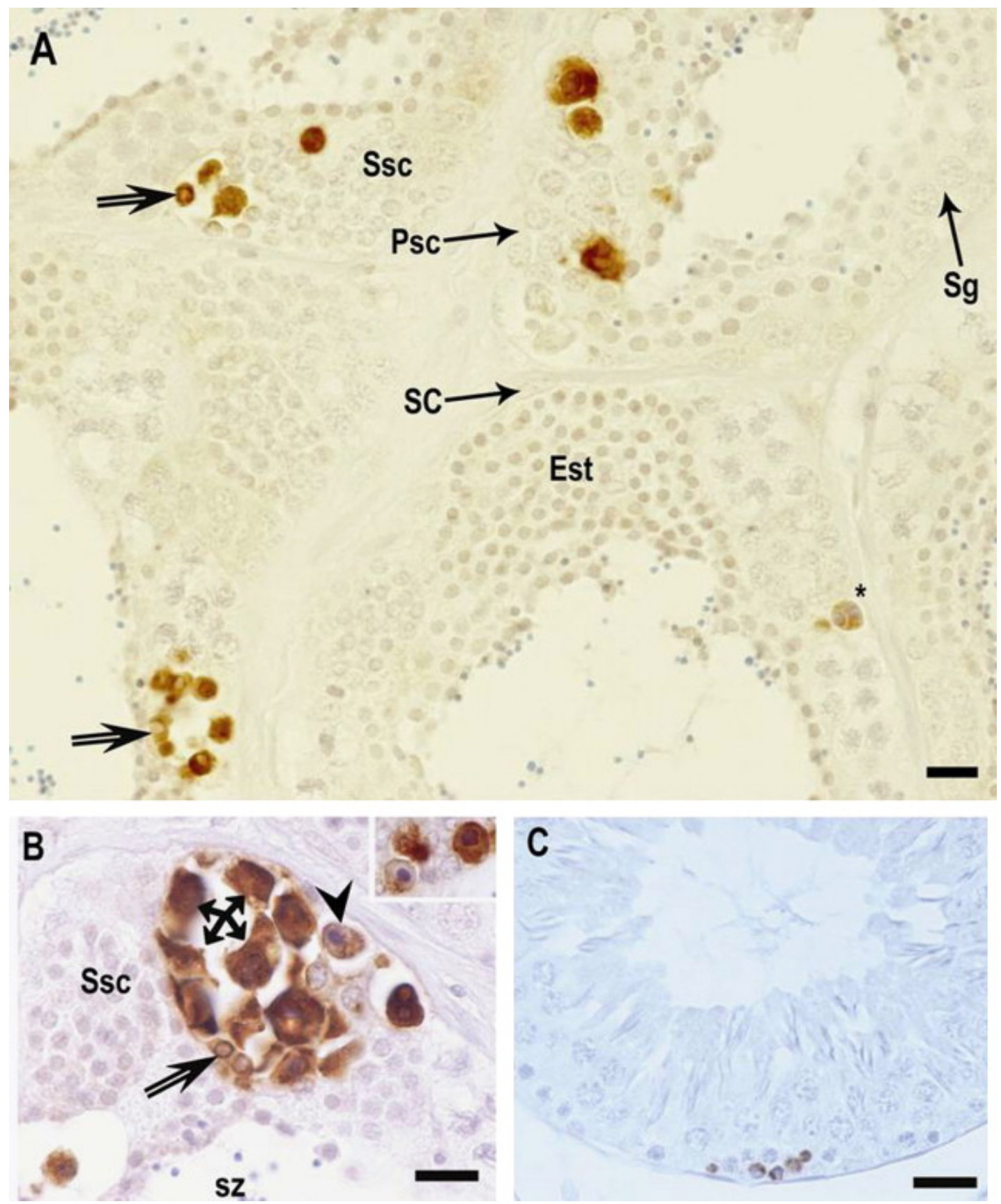

Fig. 3. Cleaved caspase-3 immunostaining patterns in spermatogenic tubules of RVD catfish. (A) Otherwise normal-looking primary and secondary spermatocyte spermatocysts often contained caspase-labelled cells, including cells in which the nuclei had a characteristic ring-like appearance (open arrow). (B) Caspase-labelling of 
nearly an entire secondary spermatocyte cyst. Some germ cells were completely and intensely labelled (arrows), whereas others clearly degenerate cells with intensely bluepurple nuclei, had diminishing caspase immunoreactivity in the cytoplasm (arrowhead) or have lost it (inset). (C) Positive control showing caspase-3-positive germ cells in pNP-treated rat testis. Sg, spermatogonia; Psc, primary spermatocytes; Ssc, secondary spermatocytes; sz, spermatozoa; SC, Sertoli cell nuclei. Bar=10 $\mu \mathrm{m}$.

Caspase-3-labelling varied intracellularly, though with a distinct pattern: labelling was either

(i) weakly cytoplasmic in cells with nuclei that had a characteristic ring-like appearance (Fig. 3A and B),

(ii) moderate to intense throughout the entire cell with the outlines of the nuclei barely discernible (Fig. 3A and B),

(iii) moderately cytoplasmic coincident with intense basophilic staining (blue) of nuclei with methyl green counterstain, such that the colour of the nuclei was identical to that of condensed chromatin in the heads of spermatozoa (Fig. 3B),

(iv) was completely lacking in such clearly degenerate cells with intensely blue-stained nuclei (Fig. 3B).

Caspase-3 immunoreactivity was also occasionally associated with germ cells at the secondary spermatocyte - early spermatid transition, such that the occasional early spermatid was also caspase-positive (not shown).

To assess whether the observed differences between TUNEL- and cleaved caspase-3immunostaining patterns may be germ cell stage and/or testicular zone-dependent, we compared the occurrences of TUNEL- and caspase-3-positive cells in the spermatogenic, mature and spent regions of RVD catfish. As shown in Fig. 4, the number of labelled cells (both caspase-3- and TUNEL-positive) varied significantly $(p<0.0001)$ according to testicular region, i.e., it was highest in the spermatogenic region, and very low in the mature and spent regions of the testis. Although the numbers of caspase-3- and TUNELpositive cells were comparable in the mature and spent tubules, the number of caspase-3positive cells in the spermatogenic tubules was three-fold higher $(p<0.05)$, higher than the number of TUNEL-positive cells (Fig. 4). Based on the multiplicity of cleaved caspase-3 immunostaining patterns observed here, results suggest that upstream cascade events during testicular apoptosis in spermatogenically active RVD catfish is prolonged and that cleaved caspase-3 immunohistochemistry is a clearly more superior method than TUNEL to quantify apoptosis. 


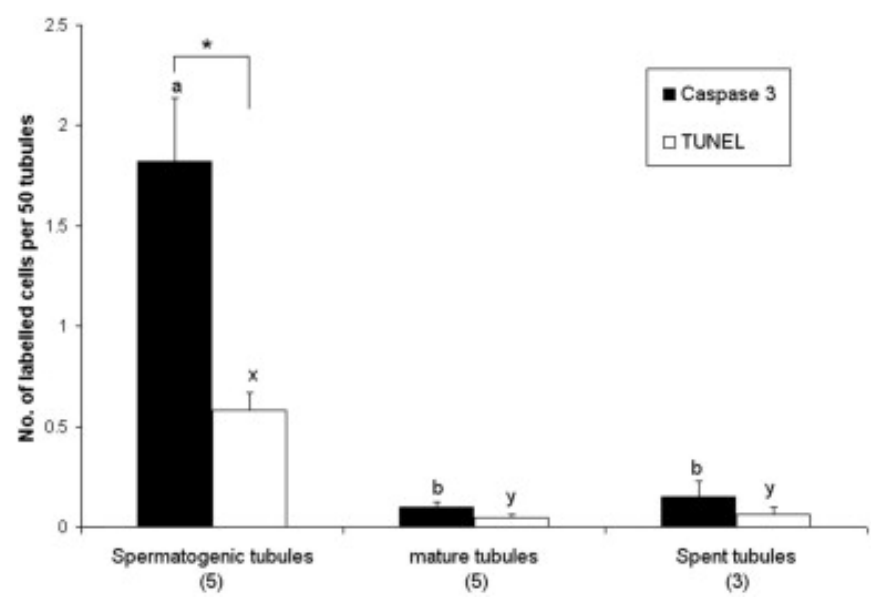

Fig. 4. Testicular zone-related changes in caspase-3-and TUNEL-labelling of germ cells in RVD catfish. Values represent the mean number \pm S.E.M. of labelled cells per 50 tubules. Animals $(n=5)$ with any given testicular zone were pooled and shown as numbers at the bottom of the figure. Differences among zones within a given method were significant $(p<0.0001)$, and is indicated with a different letter (caspase-3, $a-b$; TUNEL, $x-y)$. Differences between methods in a given zone are indicated with asterisk (*) $(p<0.05)$.

\section{Discussion}

Testicular apoptosis is described here for the first time in C. gariepinus. Cleaved caspase3 is the major executioner of apoptotic nuclear changes, and these ultimately lead to low molecular weight DNA fragmentation, which becomes TUNEL-detectable. Our findings show, however, that the number of caspase-3-positive cells in the spermatogenic region was at least three-fold higher than the number of TUNEL-positive cells, indicating that the TUNEL method underestimated the rate of testicular apoptosis in the RVD catfish. Similar discordances between TUNEL and caspase-3 immunostaining patterns were reported in ovarian follicles in buffalo and cattle (Feranil et al., 2005), and humans (Hurst et al., 2006), but these are rarely fully discussed. Using the intense blue-stained chromatin in the heads of spermatozoa as an internal control, our findings show that after intense immunoreactivity in the entire cell, caspase- 3 immunoreactivity diminishes in the cytoplasm, at which point the condensed state of the chromatin (condensed into a single mass) is revealed. Germ cells with such condensed nuclei are TUNEL-negative, whereas apoptotic cells that are TUNEL-positive lack any definitive nuclear material. These observations are consistent with a similar finding in testes of $p$-NP-treated rats (McClusky et al., 2007). Whether these phenomena are $p$-NP-induced, is not known, but they suggest that chromatin condensation and DNA fragmentation are not coincident, which agree with the notion that chromatin condensation and internucleosomal DNA fragmentation are separate events, the latter occurring at the very end of apoptosis (Robertson et al., 2000). Although low molecular weight DNA fragmentation (usually 
TUNEL-detected) in many cultured mammalian cells has been shown to be coincident with advanced chromatin margination, called stage II chromatin condensation (Samejima et al., 2001; Yuste et al., 2005), we contend that the discordances reported here for the two methods are biologically significant in vivo. One explanation for these differences may be that chromatin condensation into a single pyknotic mass, and not chromatin margination of multiple large masses which was never observed, may be specifically associated with a prolonged duration of apoptosis, which is known for other fish species as well ([McClusky, 2005] and [McClusky, 2006]). Interestingly, pyknosis induced by slow death in avian erythrocytes show no involvement of nucleases, and thus presumably no massive DNA fragmentation (Burgoyne, 1999). Secondly, it was suggested more than a century ago that the pyknotic state of chromatin or clumping of chromatin in dying neurons was followed by diffusion or ejection of DNA from the nucleus through the nuclear membrane (Collin, 1906-1907 in Clarke, 1990), which agree with the observed release into the cytoplasm of increased amounts of fragmented DNA during late apoptosis, in cultured hepatocytes (Oberhammer et al., 1996).

Based on the various nuclear changes associated with cleaved caspase-3-immunostaining patterns observed here, a sequence of the progression of germ cell apoptosis may be delineated. The initial onset of the apoptotic process in this teleost is marked by the appearance of cleaved caspase-3 immunoreactivity in the germ cell cytoplasm, coincident with the appearance of a thin layer of chromatin next to the nuclear periphery, such that the nucleus has a ring-like appearance at the light microscopic level, much like the stage I chromatin condensation in cultured mammalian cells (Samejima et al., 2001; Yuste et al., 2005). Next, cytoplasmic caspase-3 immunoreactivity increases greatly in the entire cell. Thereafter, caspase- 3 is again immunolocalised to the cytoplasm, upon which the single mass of nuclear material stains intense blue-purple with the methyl green counterstain, a colour reaction exactly similar to that of the condensed chromatin in the heads of the spermatozoa. The apoptotic cell with its condensed chromatin then gradually loses its cytoplasmic caspase-3 immunoreactivity, until it is distinctly caspase-3-negative. Finally, DNA is degraded and fragments are released into the cytoplasm, whereupon these are TUNEL-labelled.

This study also shows different pathways of cellular death in the teleost testis. Besides its involvement in the death of isolated germ cells, caspase-3 also mediates germ cell coalescence. Unlike in many other mammalian tissue systems, coalescence of germ cells is a common form of apoptotic death in the testes of fishes (McClusky, 2005). Given the intimate environment in a cyst, it may be that the cytoplasmic bridges joining germ cells, known to be at least present in another catfish (Batlouni et al., 2005), open up with resultant coalescence of adjacent cells provided the apoptotic stimulus is large enough. Conversely, a temporary interruption in cytoplasmic continuity (e.g., by way of the appearance of multiple transverse cisternae in the intercellular bridges (Dym and Fawcett, 1971)) among clone members may prevent the spread of abortive or death signals to the entire germ cell clone if the magnitude of the apoptotic signal is sub threshold within the cyst. Indeed, it has been proposed that inhibitors of apoptosis proteins (IAPs) control the threshold of caspase activation that needs to be exceeded if a cell is supposed to undergo apoptosis (Stucki and Simon, 2005). Such local control might explain the observations of 
scattered isolated apoptotic cells in otherwise healthy spermatocysts and the coincidence of apoptotic and mitotic activities in the same syncitially linked germ cell clone in $C$. gariepinus (this study) and in the shark (McClusky, 2005).

\section{Conclusion}

In summary, the results of this study showed that based on the nuclear changes accompanying its immunoexpression patterns, activated caspase- 3 has a clear and defined role in the progression of germ cell apoptosis in spermatogenically active catfish testis. Caspase-3 activation, and not TUNEL-detected DNA fragmentation, is associated with condensation of the chromatin into a single mass. These apoptotic events occur predominantly in secondary spermatocytes, germ cell stages that are known to be exquisitely androgen-sensitive. Since labelling incidences of TUNEL and cleaved caspase- 3 immunohistochemistry in the mature and spent tubules zones was very low and showed no differences between the two methods, it is concluded that these two zones are not the most suitable zones for quantification of testicular cell death ratios. The findings of this study will form the basis of future endeavours in which C. gariepinus and other teleost species will serve as a sentinel species to study the effects of endocrine disruptors on male reproduction.

The study sponsors played no role in neither the study design, in the collection, analysis, or interpretation of data, nor in the writing of the report, nor in the decision to submit the paper for publication.

\section{Conflict of interest statement}

There are no conflicts of interests.

\section{Role of the funding source}

The study sponsors played no role in neither the study design, in the collection, analysis, or interpretation of data, nor in the writing of the report, nor in the decision to submit the paper for publication.

\section{Acknowledgments}

Financial support from the National Research Foundation of South Africa (Project no. NRF 9657) and the Water Research Commission of South Africa (K5/1505) is acknowledged. The authors wish to express their appreciation to Joey Breedt of the Department of Veterinary Pathology, Faculty of Veterinary Sciences, University of Pretoria, for embedding of the tissues and tissue sectioning, and Mr. Alan Hall of the Laboratory for Microscopy and Microanalysis, University of Pretoria for assistance with the digital photomicrography. 


\section{References}

Barnhoorn, I.E., Bornman, M.S., Pieterse, G.M., Van Vuren, J.H., 2004. Histological evidence of intersex in feral sharptooth catfish (Clarias gariepinus) from an estrogen-polluted water source in Gauteng, South Africa. Environ. Toxicol. 19, 603-608.

Batlouni, S.R., Carreno, F.R., Romagosa, E., Borella, M.I., 2005. Cell junctions in the germinal epithelium may play an important role in spermatogenesis of the catfish P. fasciatum (Pisces, Siluriformes). J. Mol. Histol. 36, 97-110.

Burgoyne, L.A., 1999. The mechanisms of pyknosis: hypercondensation and death. Exp. Cell Res. 248, 214-222.

Cavaco, J.E.B., Lambert, J.G.D., Schulz, R.W., Goos, H.J.Th., 1997. Pubertal development of male African catfish, Clarias Gariepinus. In vitro steroidogenesis by testis and interregnal tissue and plasma levels of sexual steroids. Fish Physiol. Biochem. $16,129-138$.

Cavaco, J.E.B., Vilrokx, C., Trudeau, V.L., Schulz, R.W., Goos, H.J.Th., 1998. Sex steroids and the initiation of puberty in male African catfish (Clarias gariepinus). Am. J. Physiol. 275, R1793-R1802.

Clarke, P.G.H., 1990. Developmental cell death: morphological diversity and multiple mechanisms. Anat. Embryol. 181, 195-213.

Dym, M., Fawcett, D.W., 1971. Further observations on the numbers of spermatogonia, spermatocytes and spermatids connected by intercellular bridges in the mammalian testis. Biol. Reprod. 4, 195-215.

Feranil, J.B., Isobe, N., Nakao, T., 2005. Apoptosis in the antral follicles of swamp buffalo and cattle ovary: TUNEL and caspase-3 histochemistry. Reprod. Domest. Anim. 40, 111-116.

Gown, A.M., Willingham, M.C., 2002. Improved detection of apoptotic cells in archival paraffin sections: immunohistochemistry using antibodies to cleaved caspase 3. J. Histochem. Cytochem. 50, 449-454.

Hurst, P.R., Mora, J.M., Fenwick, M.A., 2006. Caspase-3, TUNEL and ultrastructural studies of small follicles in adult human ovarian biopsies. Human Reprod. 21, 1974-1980.

Labat-Moleur, F., Guillermet, C., Lorimier, P., Robert, C., Lantuejoul, S., Brambilla, E., Negoescu, A., 1998. TUNEL apoptotic cell detection in tissue sections: critical evaluation and improvement. J. Histochem. Cytochem. 46, 327-334. 
McClusky, L.M., 2005. Stage and season effects on cell cycle and apoptotic activities of germ cells and Sertoli cells during spermatogenesis in the spiny dogfish (Squalus acanthias). Reproduction 129, 89-102.

McClusky, L.M., 2006. Stage-dependency of apoptosis and the blood-testis barrier in the dogfish shark (Squalus acanthias): cadmium-induced changes as assessed by vital fluorescence techniques. Cell Tissue Res. 325, 541-553.

McClusky, L.M., de Jager, C., Bornman, M.S., 2007. Stage-related increase in the proportion of apoptotic germ cells and altered frequencies of stages in the spermatogenic cycle following gestational, lactational and direct exposure of male rats to p-nonylphenol. Toxicol. Sci. 95, 249-256.

Oberhammer, F., Froschl, G., Tiefenbacher, R., Inayat-Hussain, S.H., Cain, K., Stopper, H., 1996. Hepatocyte death following transforming growth factor-b1 addition. Microsc. Res. Tech. 34, 247-258.

Robertson, J.D., Orrenius, S., Zhivotovsky, B., 2000. Review: nuclear events in apoptosis. J. Struct. Biol. 129, 346-358.

Samejima, K., Tone, S., Earnshaw, W.C., 2001. CAD/DFF40 nuclease is dispensable for high molecular weight DNA cleavage and stage I chromatin condensation in apoptosis. J. Biol. Chem. 276, 45427-45432.

Schulz, R.W., Lubberink, K., Zandbergen, M.A., Janssen-Dommerholt, C., Peute, J., Goos, H.J.Th., 1996. Testicular responsiveness to gonadotropic hormone in vitro and Leydig and Sertoli cell ultrastructure during pubertal development of male African catfish (Clarias gariepinus). Fish Physiol. Biochem. $15,243-254$.

Stucki, J.W., Simon, H.-W., 2005. Mathematical modeling of the regulation of caspase-3 activation and degradation. J. Theor. Biol. 234, 123-131.

Yuste, V.J., Sa'nchez-Lo' pez, I., Sole' , C., Moubarak, R.S., Bayascas, J.R., Dolcet, X., Encinas, M., Susin, S.A., Comella, J.X., 2005. The contribution of apoptosisinducing factor, caspase-activated DNase, and inhibitor of caspase-activated DNase to the nuclear phenotype and DNA degradation during apoptosis. J. Biol. Chem. 280, 35670-35683.

Fig. 4. Testicular zone-related changes in caspase-3-and TUNEL-labelling of germ cells in RVD catfish. Values represent the mean number7S.E.M. of labelled cells per 50 tubules. Animals (n 1/4 5) with any given testicular zone were pooled and shown as numbers at the bottom of the figure. Differences among zones within a given method were significant (po0.0001), and is indicated with a different letter (caspase-3, a-b; TUNEL, $\mathrm{x}-\mathrm{y}$ ). Differences between methods in a given zone are 
indicated with asterisk (*) (po0.05).Chem. 280, 35670-35683.

Funding: This project was funded by the National Research Foundation of South Africa (Project no. NRF 9657) and the Water Research Commission of South Africa (K5/1505).Etical Approval: The protocol was approved by the Animal Use and Care Committee of the University of Pretoria. 